\title{
Development a New Intelligent Mobile Robot to Avoid Obstacle
}

\author{
*HUSSEIN S. Mohammed Hassan S. Mohammed Hiba A. kadim \\ Department of Computer Engineering Techniques, Alkunoozu University College, Basra, Iraq
}

\begin{abstract}
The project is a robot that automatically by passes barriers to reach a specific goal with an ultrasonic help that senses obstacles and measures the remaining transitions before the collision is meet. The robot changes its course with a couple of DC motors, Robot runs automatically without any interference by the Proportional-IntegralDerivative (PID) algorithm. The goal of this paper is to develop a path planning method that is capable of planning the mobile robot path from the starting position to the target position in different environments. However, the parameters of membership functions and PID controller parameters have optimized by using particle swarm optimization (PSO) algorithm. In addition to that, the proposed method with two Schemes of motion controllers are test with varying static and dynamic environments with and without load. The artificial potential field algorithm is introduce for path planning of mobile robot. However, the potential field algorithm is effective in avoiding unknown obstacles, but it contains minimal local problems, then a modified field algorithm is introduce to overcome some of the local minimum problems in the environment. Therefore, it is enhancing the performance of potential field algorithm and to produce a more efficient path planning method, that to allow mobile robot to navigate in dynamic and complex environments. As well as, simulation of mobile robot is design to test and implement the proposed method and control schemes using MATLAB and the software is develops by using $\mathrm{C}++$ language and Arduino IDE.
\end{abstract}

DOI: $10.7176 / \mathrm{CEIS} / 10-3-03$

Publication date: April $30^{\text {th }} 2019$

\section{INTRODUCTION}

Robotics is interested in studying those machines that can replace humans in the task of performing physical activity and decision-making. Robots are one branch of artificial intelligence. Robots now used in many fields of life such as in factories to perform high-precision jobs such as riveting and welding. Robots also used in service applications where they require extensive, independent movement capabilities, especially in situations that are harmful and dangerous to humans, for example, in defusing bombs or cleaning toxic waste. Despite the great progress that made in the field of robots over the past decade, robots are still not very useful in everyday life, because they are very clumsy in performing normal household tasks. Actually, there are many difficulties that robots may face in their environments this is due to the fact that real environment is generally, unknown, uncertain and dynamic; so, the knowledge which is available a priori on these environments may be non-existent and incomplete. From the engineering point of view, it must be independent mobile robots be flexible and good design adaptable enough to control their actions, and must be able to perform the functions required precision and accuracy. The navigation is one of the most important task, which is define as the task of following the motion or movement of a body from one position to another with respect to land-based navigation. Mobile robot's navigation can considered as a task of finding or determining a collision free path that enables the robots to transfer through obstacles from start location to a goal location. However, the mobile robot navigation problem can be classifies in the following two problems: The first one is goal access and it is a global problem because the short paths to point three a public destination, cannot obtain local information only. The topology of the environment is essential in finding good routes to the goal. The second one is "Avoiding obstacles" problem, this can be often solved using only local information, but for environments that are unknown it cannot be solved in advance because the robot needs to sense the obstacles before it can be expected to avoid them. Artificial potential field is one of the methods that used to solve above two problems of navigation, "Reaching to the goal" using attractive force of potential filed and "Avoiding obstacles" using repulsive of potential field.

\section{INTEGRAL ACTION}

The primary shortcoming of the $\mathrm{P}$ controller, tolerance of DC error, it is readily corrected by adding an integral gain to the control law. Because the integral will grow ever larger with even small DC error, any integral gain (other than zero) will eliminate DC droop. This single advantage is why PI is so often preferred over P control. Integral gain provides DC and low-frequency stiffness. Stiffness is the ability of a system to resist disturbances. Integral gains operate in the low-frequency zone and so improve stiffness at relatively low frequencies (those well below the bandwidth). When a DC error occurs, the integral gain will move to correct it and the higher the integral gain, the faster the correction. The third gain that used for controllers is the differential, or "D," gain. The D gain advances the phase of the loop by virtue of the $90^{\circ}$ phase lead of a derivative. Using D gain will usually allow the system responsiveness to increase, for example, allowing the bandwidth to nearly double in some cases. Derivative 
action is normally introduce to increase the damping in the system and amplifies the existing noise, which can cause instability. Differential gain has shortcomings. Derivatives have high gain at high frequencies. So, while some D does help the phase margin, too much hurts the gain margin by adding gain at the phase crossover, typically a high frequency. This makes the $\mathrm{D}$ gain difficult to tune. It may at once reduce overshoot by virtue of increased PM (phase margin) and cause high-frequency oscillations owing to the reduced GM (gain margin). The highfrequency problem is often hard to see in the time domain because high frequency ringing can be hard to distinguish from normal system noise. Therefore, a control system may be accept at installation but have marginal stability and thus lack the robust performance expected for factory equipment. This problem is much easier to see using Bode plots measured on the working system. Another problem with derivative gain is that derivatives are sensitive to noise. Even small amounts of noise from wiring or resolution limitations may render the D gain useless. In most cases, the D gain needs to follow by a low-pass filter to reduce the noise content.

\section{MOTOR WITH PROPORTIONAL-INTERGRAL}

Integral gain comes at a cost. PI controllers are more complicated to implement; the addition of a second gain is part of the reason. In addition, dealing with power converter saturation becomes more complicated. In analog controllers, clamping diodes must added; in digital controllers, special algorithms must encode. The reason is that the integral must clamped during saturation to avoid the problem of "wind-up". Integral gain also causes instability. The common result of adding integral gain is increased overshoot and ringing. Before leave the discussion of integrators, there are two more things, it need to point out. First, since you are adding up the error over time, the sampling time that you are running becomes important. Second, you need to pay attention to the range of your integrator to avoid wind-up.

\section{Motor with PI control and windup}

If you have, a controller that needs to push the plant hard your controller output will spend significant amounts of time outside the bounds of what your drive can actually accept. This condition is call saturation. If you use a PI controller, then all the time spent in saturation can cause the integrator state to grow (wind up) to very large values. When the plant reaches the target, the integrator value is still very large, so the plant drives beyond the target while the integrator unwinds and the process reverses. This situation can get so bad that the system never settles out, but just slowly oscillates around the target position.

\section{PID CONTROLLER FUNDAMENTALS}

The input/output relation for an ideal PID controller with error feedback is.

$u=K_{p} e+K_{i} \int_{0}^{t} e(\tau) d \tau+K_{d} \frac{d e}{d t}=K_{p}\left(e+\frac{1}{T_{i}} \int_{0}^{t} e(\tau) d \tau+T_{d} \frac{d e}{d t}\right)$

It can find transfer function of the Proportional-Integral-Derivative controller in the Laplace domain.

$$
C(S)=K_{p}+\frac{K_{i}}{S}+K_{d} S
$$

The control action is thus the sum of 3 terms; proportional feedback, the integral term and the derivative action. For this reason, PID controllers were originally call three term controllers. The controller parameters are the proportional gain $\mathrm{KP}$, the integral gain $\mathrm{Ki}$ and the derivative gain $\mathrm{Kd}$. The time constants $\mathrm{Ti}$ and $\mathrm{Td}$, called integral time (constant) and derivative time (constant), are sometimes use instead of the integral and derivative gains as shows in figure 1.

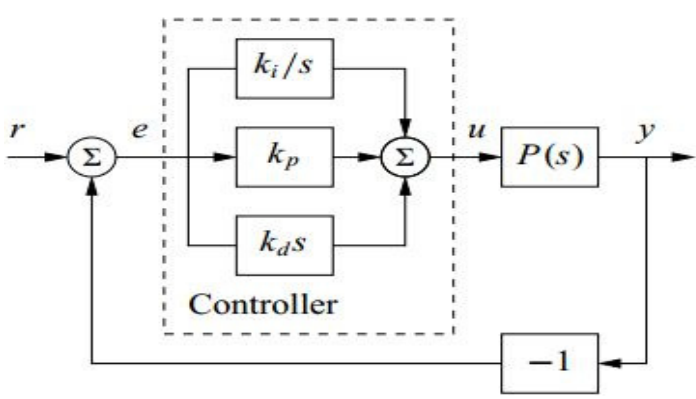

Figure 1. Proportional-Integral-Derivative using error feedback in the Laplace (S) domain.

\section{PID Controller}

Using a Proportional-Integral-Derivative control-er is to apply a sum of three types of control actions: the relative procedure, the integral action, and the derivative work that they will describe separately. The proportional action Proportional control is the easiest feedback control to implement, and simple proportional control is probably the most common kind of control loop. A proportional controller is just the error signal multiplied by a constant and 
fed out to the drive. It is a major step forward to let the magnitude of the corrective action depends on the magnitude of the error. This has the effect that a small error will lead to only a small adjustment, whereas a larger error will result in a greater corrective action. The simplest way to achieve this effect is to let the controller output be proportional to the tracking error:

Where $\mathrm{kp}$, the controller gain, is a positive constant.

$$
\mathrm{U}_{\mathrm{p}}(\mathrm{t})=\mathrm{K}_{\mathrm{P}} \mathrm{e}(\mathrm{t})
$$

\section{THE PLATFORM}

The robot used in this experiment contains a differential motor (two motors) with an ultrasonic sensor installed on the servomotor in the front part of the robot and its detection of obstacles. Figure (2) shows the robot building.

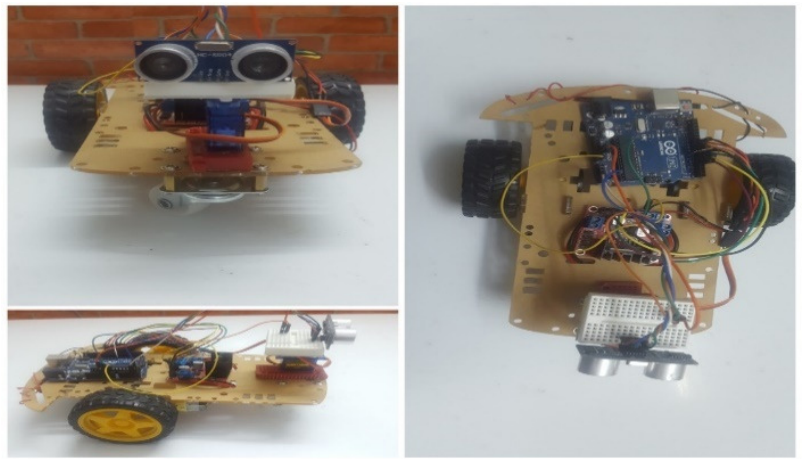

Figure 2. Robot structure used.

\section{HARDWARE COMPONENTS}

The components that must be equipped for the robot are the sensors and wires and a precise control unit used to read the data from the sensors and send the commands to the motor to reach the desired target.

\section{CONTROLLING DC MOTORS}

To control two DC motors of mobile robot, first it is connected each motor to the A(Out 1 \& Out 2) and B (Out 3 \& Out 4), Next, we powered module by connecting it with power supply 16v (Battery), After that the positive end goes to Pin 5V and the negative side to Pin GND. Then, we connect the Arduino digital output pins to the driver module, the digital pins (4,5,6 and 7) of Arduino will be connected to pins IN1, IN2, IN3 and IN4 of L298N driver respectively, the following figure (3) illustrates the method of linking.

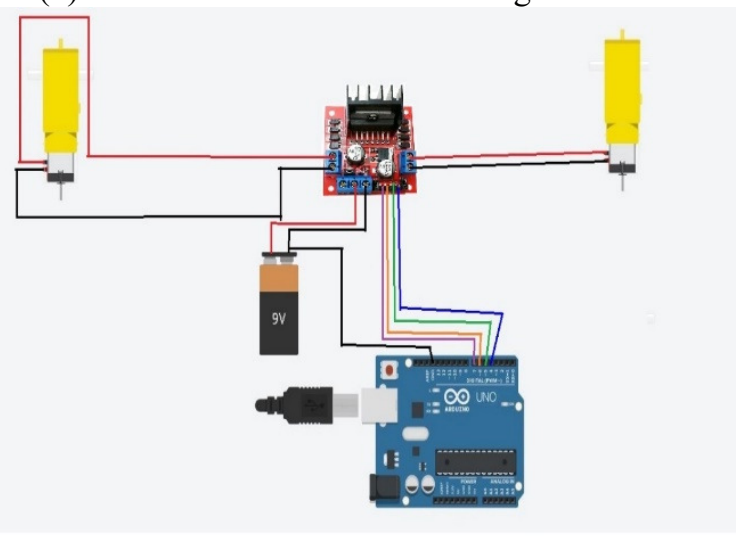

Figure 3. DC motors configuration scheme.

The direction of motor is control by sending a HIGH or LOW signal to the drive for each motor. For instance, for first motor, a LOW to IN1 and a HIGH to IN2 will cause it to turn in one direction, and a HIGH and LOW will cause it to turn in the reverse direction.

\section{CONNECTION OF ULTRASONIC SENSOR}

We connect one sensor to the robot at a 180-degree angle, there are 4 pins for the sensor, from left to right, first pin is Vec, second pin is Trig (Trigger input pin), third pin is Echo (Receiver output pin) and four pins is GND pin. Trig pin is connect to A4 pin in Arduino board and echo pin is connect to A5 pin in Arduino board. The following figure (4) illustrates the method of linking. 


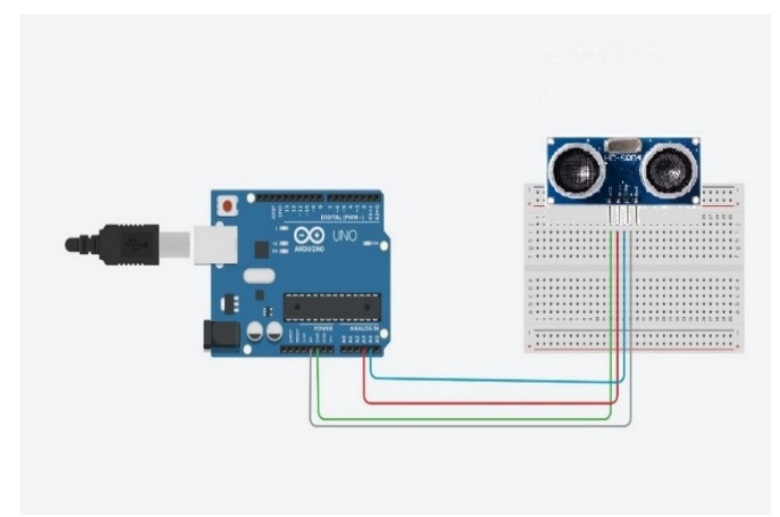

Figure 4. ultrasonic configuration scheme.

\section{CONNECTION SERVO MOTOR}

It used the servomotor and the ultrasonic in such a way that the servomotor should control the direction of the ultrasonic sensor from $0^{\circ}$ to $180^{\circ}$. The servomotor has three connection pins, these pins are brown, red and orange which are connect to Arduino board pins (10,9 and 8) respectively, the following figure (5) illustrates the method of linking.

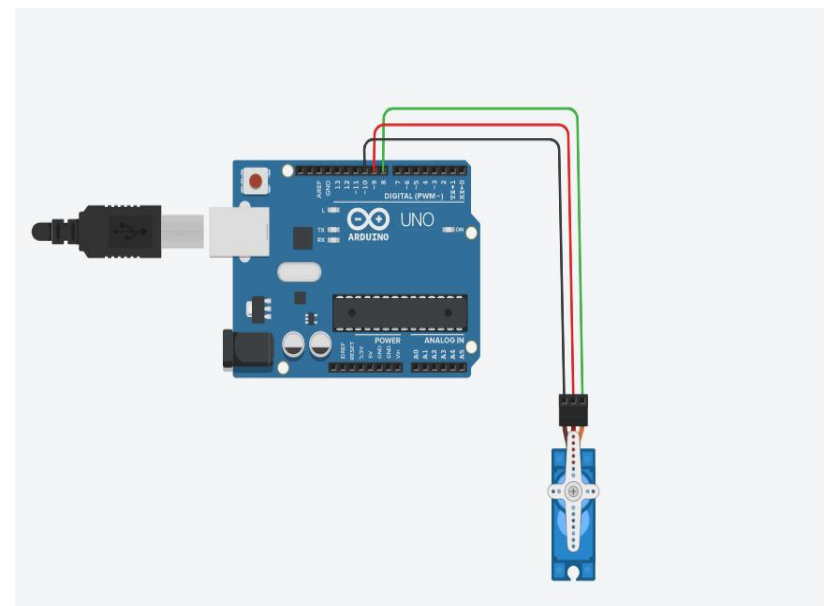

Figure 5. Servo motor configuration scheme.

\section{IMPLEMENTATION OF THE FORCES OF POTENTIAL FIELD}

In real world, the robot is give an initial position start and a goal position goal, the artificial potential field method is then constantly calculated and updated as the mobile robot faces obstacles in the environment. The mobile robot must compute attractive and repulsive forces of artificial potential field that acting on mobile robot. As well as, it is need to compute the attractive force, the distance between mobile robot and goal. However, should determine, since the goal of position is fix then the coordinate of goal. In addition to that, it is known in the environment, the position of mobile robot is record with odometer and encoders, so the positions of mobile robot and goal are known then the distance between mobile robot and goal could be determined and then the attractive force.

\section{ROBOT TEST}

To prove that the artificial potential field algorithm works as desired the mobile robot is test through several experiments in environments have different configuration of obstacles. The real environment of mobile robot is consider as Cartesian coordinate system. The first test in the environment that contains three obstacles with configuration as show in Figure (6). The test shows that mobile robot can avoid obstacles successful and reach approximately to the goal point. 


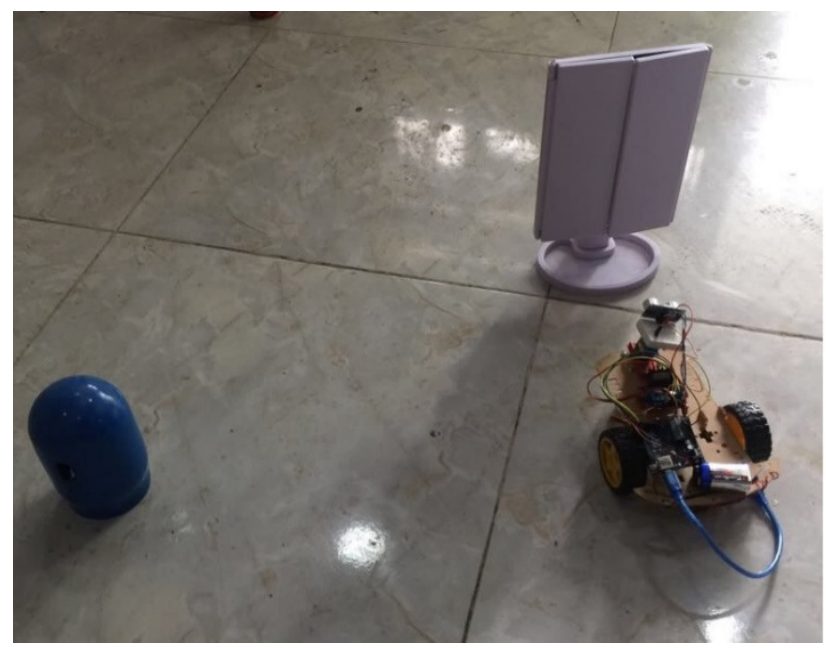

Figure 6 . environment with three obstacles.

\section{CONCLUSION}

In this paper, it was propose a new robot that automatically by passes barriers to reach a specific goal with an ultrasonic help that senses obstacles and measures the remaining transitions before the collision is meet. As well as, it is enhancing the performance of potential field algorithm and to produce a more efficient path planning method, to allow mobile robot to navigate in dynamic and complex environments. However, the results were $100 \%$ successful and the robot skipped all the barriers that interface in its specified path and reached the goal in both tests. To make sure more of the work of the robot we have changed the places of barriers and change course from the first experience and then the robot reached the goal and avoid all obstacles successful.

\section{REFERENCES}

Chang, Y., Yamamoto, Y. "Path planning of wheeled mobile robot with simultaneous free space locating capability". Intelligent Service Robotics, Vol. 2(1), pp.9-22, 2008.

Sirkka Liisa, Jämsä Jounela . "Simulation study of self-tuning adaptive control for rougher flotation". Powder Technology, Vol. 69(1), pp.33-46, 1992.

Pandey, A., Pandey, S., Gupta, P. "Intellige-nt navigation and control of a mobile robot in static and dynamic environments using hybrid fuzzy architecture". International Journal of Autonomic Computing, Vol. 2(3), p.255, 2017.

Dong, X., Jian-qu, Z., Feng, W. "Fuzzy PID Control to Feed Servo System of CNC Machine Tool". Procedia Engineering, Vol. 29, pp.2853-2858, 2012.

M. Yilmaz. "2BA BEDEN BEYİN AKIM-I'NDAKİ POSTMODERNİST UNSU-RLAR". Journal of Turkish Research Institute, Vol. (55), pp.345-345, 2016.

Al-Assadi, S. "An approach for pole assignent by dynamic compensator with pres-pecified poles". Applied Mathematical Modelling, 20(2), pp.135-142, 1996.

D. Mohammed Abdul Ahad. "Low Cost Obst-acle Avoidance Robot with Logic Gates and Gate Delay Calculations". Automation, Control and Intelligent Systems, Vol. 6(1), p.1, 2018.

FERGUSON, N. "Keynote 3 Nonlinear systems for vibration control (The 12th International Conference on Motion and Vibration Control)". The Proceedings of the Symposium on the Motion and Vibration Control, 2014.12(0), pp._Keynote3--_Keynote3-, 2014.

YANG, T. "Formation Control and Obstacle Avoidance for Multiple Mobile Robots". Acta Automatica Sinica, Vol. 34(5), pp.588-592, 2008.

Janert, P., Schick, M. "Firs t- order unbinding and preunbinding in amphiphilic systems". Physical Review E, Vol. 54(1), pp. R33-R35, 1996.

HE, L. "Activity of Serine / Threonine Protein Phosphatase Type-2C (PP2C) and Its Relationships to Drought Tolerance in Maize". ACTA AGRONOMICA SINICA, Vol. 34(5), pp.899-903, 2008.

YANG, J. "An Analytical Design Method of PID Controller Based on AQM / ARQ". Journal of Software, 17(9), p.1989, 2006.

Gonzalez, J., Ollero, A., Hurtado, P. "Local Map Building for Mobile Robot Autonomous Navigation by Using a 2D Laser Range Sensor". IFAC Proceedings, Vol. 26(2), pp.835-838, 1993.

Trahair, N. "Guide to stability design criteria for metal structures (4th edition)". Engineering Structures, 11(3), p.202, 1989. 